\title{
The type three secretion system facilitates migration of Burkholderia terrae BS001 in the mycosphere of two soil-borne fungi
}

\author{
Pu Yang ${ }^{1} \cdot$ Miaozhi Zhang ${ }^{1} \cdot$ Jan Aaldrik Warmink ${ }^{1} \cdot$ Miao Wang ${ }^{2} \cdot$ \\ Jan Dirk van Elsas ${ }^{1}$
}

Received: 12 May 2016 / Revised: 10 July 2016 / Accepted: 23 July 2016 /Published online: 12 August 2016

(C) The Author(s) 2016. This article is published with open access at Springerlink.com

\begin{abstract}
The type three secretion system (T3SS) is known to play a critical role in several bacterial-eukaryotic cell interactions. Recent indirect evidence has also pointed to a role of this system in bacterial-fungal interactions in soil. In the current study, we examine if the T3SS of the fungal-interactive Burkholderia terrae strain BS001 can aid in the interaction of this bacterium with two soil fungi, i.e., Lyophyllum sp. strain Karsten and Trichoderma asperellum 302. We first analyzed the T3SS of strain BS001 and then constructed a knockout mutant of the essential sctD gene. The selected $s c t D$ mutant strain did not show any differences to the wild-type strain with respect to its growth and nutrient utilization behavior, excluding polar effects of the mutation. Then, the migration ability of the sctD mutant strain along with the hyphae of Lyophyllum sp. strain Karsten growing through presterilized soil was tested, revealing hampered comigration as compared to the wildtype strain. The effect was also observed with $T$. asperellum 302. However, the migration impairment was only noticed in mixed-inoculation experiments, whereas it remained unnoticed when the two strains were inoculated in separate. These data demonstrate that the T3SS assists $B$. terrae BS001 in its interaction with two soil fungi, without being essential for these interactions. As far as we know, this is the first
\end{abstract}

$\mathrm{Pu}$ Yang

p.yang@rug.nl

1 Microbial Ecology, Groningen Institute for Evolutionary Life Sciences, University of Groningen, Nijenborgh 7, 9747 AG Groningen, The Netherlands

2 Microbial Community Ecology, Groningen Institute for Evolutionary Life Sciences, University of Groningen, Nijenborgh 7, 9747 AG Groningen, The Netherlands time that the role of a T3SS in the comigration of bacteria along with soil-exploring fungi is verified directly.

Keywords Burkholderia terrae BS001 - Bacterial migration · Type 3 secretion system $\cdot$ Mycosphere $\cdot$ Bacterial-fungal interaction

\section{Introduction}

The type three secretion system (T3SS) is a protein delivery system in Gram-negative bacteria, which is divided into two families, i.e., (1) the flagellar and (2) the non-flagellar T3SS (NF-T3SS). The former system drives motility, and the latter is often involved in pathogenesis (Abby and Rocha 2012). In the subsequent text, we prefer to use the term T3SS (instead of NF-T3SS), as we place a focus on the NF-T3SS in this paper. The T3SS system is very complex, consisting of more than 20 proteins (Galan et al. 2014). These include extracellular components, the so-called outer membrane ring complex and an inner membrane ring complex (Yip and Strynadka 2006). The T3SS is located at the bacterial inner membrane and spans this, as well as the outer membrane, reaching out to the exterior on one side and the host cell cytoplasm on the other. It has been reported that the T3SS mediates the attachment of bacterial cells to plant or animal cell surfaces and the injection of effector proteins into the cytosol of host cells. Thus, the T3SS is involved in multiple bacterial-eukaryotic interactions that range from symbiosis (Silver et al. 2007; Correa et al. 2012), particularly in nitrogen-fixing bacterialplant interactions (Dai et al. 2008; Okazaki et al. 2009; Saad et al. 2012; Piromyou et al. 2015) to parasitism, especially in bacterial-animal interactions (Plano and Schesser 2013; Neeld et al. 2014). Thus, T3SSs play diverse roles in the interactions of bacteria with their hosts. 
Additionally, several previous studies have suggested a role for the T3SS in bacterial-fungal interactions. Warmink and van Elsas found that the abundance of T3SS-positive isolates was dramatically higher in the mycosphere of Laccaria proxima than in corresponding bulk soil (Warmink and van Elsas 2008). Another study showed that T3SS-positive pseudomonads were significantly more abundant on mycorrhizal roots than on non-mycorrhizal ones (Viollet et al. 2011). Also, the intracellular bacterium Burkholderia rhizoxinica was found to employ its T3SS in the interaction with the fungal host Rhizopus microsporus (a pathogen of rice), modulating sporulation (Lackner et al. 2011). On the other hand, the T3SS can be involved in antagonistic bacterium-host interactions, as revealed by the fact that the biocontrol activity of Pseudomonas fluorescens against the cucumber pathogen Pythium ultimum was strongly reduced when a T3SS-deficient mutant strain was used instead of the wild-type (Rezzonico et al. 2005). Remarkably, mutation in the T3SS locus did not affect the growth-promoting effect of another Pseudomonas fluorescens, the mycorrhization helper strain BBc6R8, on Laccaria bicolor in vitro. However, it failed to promote the establishment of the Douglas fir-L. bicolor symbiosis (Cusano et al. 2011).

Burkholderia terrae BS001, an excellent colonizer of fungal hyphae (Nazir et al. 2014), can migrate along with growing hyphae of Lyophyllum sp. strain Karsten, as well as Trichoderma asperellum 302 and Fusarium oxysporum Fo47. It can form biofilms around the hyphae of all of these fungi (Warmink and van Elsas 2009; Nazir et al. 2014). Migration along with Lyophyllum sp. strain Karsten was found in various soils (Nazir et al. 2012). Strain BS001 also facilitated the movement of the non-migrator Dyella japonica BS013, allowing it to disperse along with the fungal hyphae (Warmink et al. 2011). Interestingly, B. terrae BS001 was able to survive in acid soil ( $\mathrm{pH} 4.1-4.5)$ only in the presence of Lyophyllum sp. strain Karsten hyphae (Warmink and van Elsas 2009). Furthermore, it protected this fungal host against antagonists in soil microcosms (Nazir et al. 2014) but inhibited primordium setting (leading to mushroom formation) in liquid microcosms (Nazir et al. 2013). Thus, the interactions between B. terrae BS001 and its fungal host are very intricate, making it likely that a plethora of mechanisms are involved.

Analysis of the genome of B. terrae BS001 recently yielded evidence for the contention that one functional T3SS gene cluster is present in this strain (Haq et al. 2014). In a previous study, molecular-based evidence suggested a role for the T3SS in the bacterial interaction with soil fungi (Warmink and van Elsas 2009). However, no direct evidence on the basis of tests with living bacteria has as yet been presented. In the current study, we further analyzed this system, hypothesizing that the T3SS of B. terrae BS001 is involved in the interaction between this organism and its host fungi in soil. To test this hypothesis, we constructed a mutant of strain BS001 with a debilitated T3SS, on the basis of the $s c t D$ gene.
We subsequently examined the effect of the mutation on the migration behavior of strain BS001 in the mycosphere of two selected soil fungi, i.e., the basidiomycete Lyophyllum sp. strain Karsten and the ascomycete T. asperellum 302. The results support the notion that the T3SS plays a non-essential role in the $B$. terrae BS001-fungal interaction in soil.

\section{Materials and methods}

\section{Strains and cultural conditions}

B. terrae $\mathrm{BS} 001$, which was isolated from the mycosphere (Warmink and van Elsas 2009), was cultured at $28^{\circ} \mathrm{C}$ in LB broth $(10 \mathrm{~g} / \mathrm{L}$ tryptone, $5 \mathrm{~g} / \mathrm{L}$ yeast extract, and $5 \mathrm{~g} / \mathrm{L} \mathrm{NaCl}$, Sigma-Aldrich Co., USA) with shaking, or on R2A agar $(0.5 \mathrm{~g} / \mathrm{L}$ yeast extract, $0.5 \mathrm{~g} / \mathrm{L}$ proteose peptone no. $3,0.5 \mathrm{~g} / \mathrm{L}$ casamino acids, $0.5 \mathrm{~g} / \mathrm{L}$ dextrose, $0.5 \mathrm{~g} / \mathrm{L}$ soluble starch, $0.3 \mathrm{~g} / \mathrm{L}$ sodium pyruvate, $0.3 \mathrm{~g} / \mathrm{L}$ dipotassium phosphate, $0.05 \mathrm{~g} / \mathrm{L}$ magnesium sulfate, and $15 \mathrm{~g} / \mathrm{L}$ agar, Difco, USA). The fungal hosts used in this study were Lyophyllum sp. strain Karsten (DSM2979) and T. asperellum 302 (Nazir et al. 2014). Fungi were grown on oat flake agar (OFA, $30 \mathrm{~g} / \mathrm{L}$ oat flake, $15 \mathrm{~g} / \mathrm{L}$ agar, Warmink and van Elsas 2009) at $28{ }^{\circ} \mathrm{C}$. Once every 4 weeks, fungal strains were transferred to fresh OFA for maintenance.

\section{Construction of an sctD mutant of B. terrae BS001}

A T3SS mutant strain was constructed by knocking out the $s c t D$ gene via double crossover allelic exchange using suicide vector pSUP101 (chloramphenicol $[\mathrm{Cm}]$ resistance, Simon et al. 1983). Figure 1 shows the strategy used. Fragments homologous to the $s c t D$ flanks were amplified by PCR using primer pairs sctD-LF1/sctD-LR and sctD-RF/sctD-RR1, respectively (Fig. 1). Primers were designed based on the published genome sequence of $B$. terrae BS001 (Haq et al. 2014) using Primer Premier 5 software. The two fragments were purified and fused by fusion PCR using primer pair sctDLF1/sctD-RR1. The fused fragment was ligated into the pGEM-T vector (Promega Corporation, Madison, USA), and the new construct was verified by agarose gel electrophoresis. Then, the construct was digested with HindIII and the relevant fragment was ligated with HindIII-digested pSUP101 DNA, yielding pSUP101- $\Delta s c t D$. Following this, vector pSUP101- $\Delta s c t D$ was introduced into the $B$. terrae BS001 genome by conjugation with the mobilizing strain Escherichia coli S17-1 (pSUP101- $\Delta$ sctD) (Simon et al. 1983), selecting a presumed single crossover mutant on R2A agar supplemented with nitrofurantoin $(50 \mathrm{mg} / \mathrm{L})$ and chloramphenicol $(25 \mathrm{mg} / \mathrm{L})$. Finally, five colonies grown on this medium were streaked to purity, after which one was transferred to fresh LB broth, grown (shaking, $28^{\circ} \mathrm{C}$ ), and transferred daily to new LB broth 
a

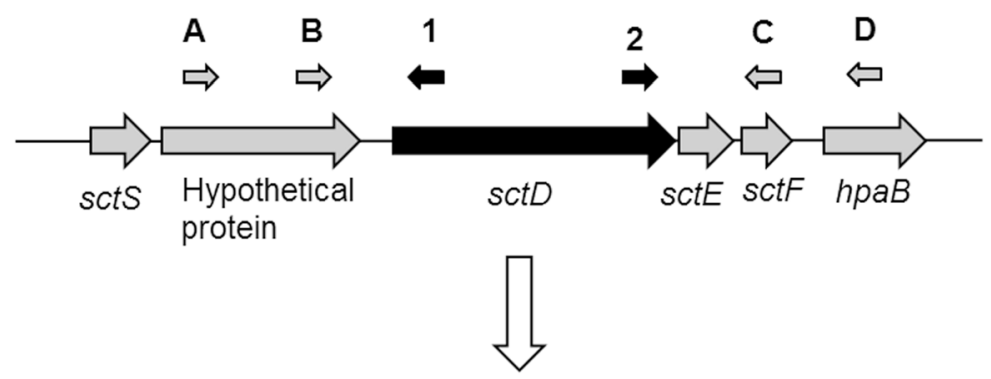

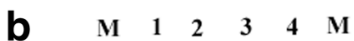



Fig. 1 Basic idea of $s c t D$ knockout. a Primer position along with $s c t D$ and the flanking genes. $A, B, C, D, 1,2$ are primers. $A$, sctD-LF2; $B$, sctDLF1; $C$, sctD-RR1; $D$, sctD-RR2; 1, sctD-LR, 2, sctD-RF. b PCR verification of $s c t D$ knockout. $M$, GeneRuler 1-kb DNA Ladder (ThermoFisher Scientific). 1, 2, used primer pair sctD-LF1/ sctD-RR1;

in order to select the (desired) double-crossover mutant strain. Following 14 transfers, the culture was diluted and spread on R2A agar without antibiotics. Single colonies were randomly picked and checked for $\mathrm{Cm}$ resistance $(25 \mathrm{mg} / \mathrm{L})$. Five $\mathrm{Cm}-$ sensitive colonies were streaked to purity. Then, primer pairs sctD-LF1/sctD-RR1 and sctD-LF2/sctD-RR2 were used on colony material to identify the desired mutant strain. For the details of the strategy and the primers used, see Fig. 1a and Table 1.

\section{Soil microcosms}

For all experiments, soil from Gieterveen, the Netherlands, was used (denoted $\mathrm{G}$ soil). The soil was freshly sampled as topsoil (10-cm depth) and taken to the laboratory, where it was homogenized. The $\mathrm{G}$ soil was characterized as a sandy loam, with $\mathrm{pH} 5.1$ and organic carbon (C) and total nitrogen $(\mathrm{N})$ contents of 2.8 and $0.8 \%$. The soil was adjusted to $\mathrm{pH} 6.8$ by adding $0.5 \% \mathrm{CaCO}_{3}$. Then, it was autoclaved $\left(121{ }^{\circ} \mathrm{C}, 27 \mathrm{~min}\right)$ three times, with intermittent incubation
3, 4, used primer pair sctD-LF2/ sctD-RR2; 1, 3, used genomic DNA of B. terrae BS001 $\Delta s c t D$ as template; 2, 4, used genomic DNA of B. terrae BS001 wild-type strain as template. The PCR product of $\Delta s c t D$ mutant strain were $1 \mathrm{~kb}$ less than wild-type strain using each primer pair, respectively

at room temperature. Soil microcosms were then prepared aseptically, in three-compartment Petri dishes according to Warmink and van Elsas (2009). Briefly, one compartment was filled with OFA, and the other two with the sterile amended G soil (moisture content $17 \%$ ).

To start up the experiments, OFA plugs containing fungal growth were placed in the OFA compartments of the microcosms, and allowed to grow out at $28^{\circ} \mathrm{C}$. At the time the fungi over the barrier between the OFA and soil compartments (approx. 5 days for Lyophyllum sp. strain Karsten and 3 days for $T$. asperellum 302 ), about $5 \times 10^{5}$ bacterial cells were introduced at the hyphal growth fronts in each soil compartment. Three different experimental setups were performed: (1) B. terrae BS001 wild-type strain alone, (2) B. terrae BS001 $\Delta s c t D$ mutant strain alone, and (3) a 1:1 mixture of wild-type and mutant strains. The soil microcosms were closed using parafilm and incubated horizontally at $28{ }^{\circ} \mathrm{C}$. Then, soil samples were recovered, by punching out at both the inoculation and the migration sites (i.e., at the hyphal fronts) at days 4, 7, and 14 for Lyophyllum sp. strain Karsten
Table 1 Primers used in this study

\begin{tabular}{lll}
\hline Primer names & Sequence $\left(5^{\prime}-3^{\prime}\right)$ & Restriction enzyme \\
\hline sctD-LF1 & GCTAAGCTTCGCCCAGGTTCGCCAGTC & Hind III \\
sctD-LF2 & GCTAAGCTTGACGGGCCGCTCCGG & Hind III \\
sctD-RR1 & GCTAAGCTTGTGACAGTGCTCAGTGCGGATG & Hind III \\
sctD-RR2 & GCTAAGCTTGAAGCAGTCGAGCCGCACAT & Hind III \\
sctD-LR & TTCGTTTGCGGCTTTCCAGTCGGTGATGCGAATGTC & \\
sctD-RF & AAAGCCGCAAACGAACCGC & \\
\hline
\end{tabular}

The restriction sites at the primers are shown by underline. The nucleotides in bold represent 15 -bp overlap sequences used for fusion PCR 
or day 2 for T. asperellum 302. The samples were suspended in $0.85 \% \mathrm{NaCl}$, shaken intensely ( $1 \mathrm{~min}$, three times, with 30 -s intervals), serially tenfold diluted and spread on R2A plates. For each experiment, four replicates were used.

\section{Analysis of strain BS001 population composition in the mixed-inoculant experiments}

The percentages of the mutant strain in the mixed culture samples were measured by colony PCR using primer pair sctD-LF1/sctD-RR1. Following incubation, 24 colonies were picked randomly from suitable-diluted R2A agar plates and subjected to PCR analysis with the above primers. The PCR products were checked by agarose gel electrophoresis, and the numbers of colonies showing either wild-type or mutant amplicon sizes were quantified.

\section{Statistical analysis of the data}

All data obtained were subjected to analysis of variance (ANOVA) using the statistical program SPSS. Differences of the means were considered to be significant at $P<0.05$.

\section{Results}

\section{Analysis of the B. terrae BS001 T3SS}

Overall information on the single T3SS in the B. terrae BS001 genome was obtained from Haq et al (2014). A more detailed analysis of this system, like performed here, revealed the system to be $18.0 \mathrm{~kb}$ in length, showing the presence of genes for 22 predicted proteins (Table 2). As suggested previously (Haq et al 2014), the T3SS of B. terrae BS001 belongs to the hrp2 family, which includes most of the Burkholderia and Ralstonia T3SSs. It is to a large extent syntenous with the T3SS system of $B$. rhizoxinica HKI 0454 (Lackner et al. 2011; Haq et al. 2014).

The predicted protein $\mathrm{HpaB}$ (Haq et al. 2014) was found to be identical to HpaB encoded by B. rhizoxinica HKI 0454 (Lackner et al. 2011). BLAST-P analysis showed that it contains a conserved domain which belongs to the Tir chaperone protein (CesT) family. CesT serves as a chaperone to maintain the stability of secreted proteins and their presentation in a secretion-competent state in the cytosol (Delahay et al. 2002). Secondly, the predicted 73 amino acid protein denoted SctF, a possible functional homologue of SctF (Haq et al. 2014), potentially represents the main component of the pilus of the T3SS injectisome (Hoiczyk and Blobel 2001), also being required in host cell detection (Torruellas et al. 2005). SctE has been proposed to be involved in needle assembly (Lackner et al. 2011); however, the exact function of this protein has not been elucidated yet. SctD is predicted to be located on the inner membrane, forming the basal body together with SctJ and SctC. Furthermore, the SctR, SctS, SctT, $\mathrm{SctU}$, and SctV proteins were predicted to be involved in formation of the main effector export apparatus (Diepold et al. 2011), linking with SctD as mediated by SctJ (Diepold et al. 2010). Furthermore, we found all other predicted T3SS proteins in the canonical order. These are SctQ (formation of cytosolic complex C-ring, Diepold et al. 2015), SctP (controlling the length of the needle; Journet et al. 2003), SctI (inner rod protein; Wood et al. 2008), SctL (combines SctQ and SctK to form a "sorting platform" that governs the order of substrate export; Jackson and Plano 2000; Diepold et al. 2015), SctN (ATPase, essential for stabilization of C-ring; Jackson and Plano 2000; Diepold et al. 2015), and $\mathrm{SctO}$ (unknown function). The last one gene was not complete since it was at the end of the contig. However, it is homologous to SctC (BLASTP).

\section{General properties of the B. terrae BS001 knockout mutant BS001 $\Delta s c t D$}

After the knockout process (see Materials and methods), $1014 \mathrm{bp}$ of the $s c t D$ gene in B. terrae BS001 was deleted. The mutant strain, which was coined $B$. terrae BS001 $\Delta s c t D$, was examined by direct PCR focusing on the $s c t D$ deletion site and revealed amplicons of around $1 \mathrm{~kb}$ smaller than those generated by the wild-type strain (two different sets of primers used, Fig. 1b). Furthermore, the mutant strain B. terrae BS001 $\Delta s c t D$ showed very similar growth dynamics to the wild-type in LB medium (Fig. 2). Additionally, we tested the phenotypic trait patterns of the two strains by BIOLOG GEN III microplates. In this analysis, the mutant strain showed exactly the same substrate utilization pattern as the wild-type strain, as detailed below. The BIOLOG GEN III plates consist of two parts, i.e., (1) 71 carbon source utilization assays (columns 1-9), and (2) 23 chemical compound sensitivity assays (columns 10-12). Concerning the results from (1), these were consistent with results reported before, using the GN2 plate (Nazir et al. 2012). With respect to (2), the wild-type and mutant strains revealed the same tolerance to low $\mathrm{pH}$, $\mathrm{NaCl}$, and particular antibiotics.

\section{Population dynamics of B. terrae BS001 in the mycosphere of Lyophyllum sp. strain Karsten}

Single strain inoculations Both the wild-type and mutant strain survived well at the inoculation site in the mycosphere of Lyophyllum sp. strain Karsten, reaching around $10^{8}$ cells $/ \mathrm{g}$ dry soil (Fig. 3a). Moreover, they were also capable of migrating through soil along with the growing fungal hyphae (Fig. 3b), reaching up to $10^{9}$ cells/g dry soil at the migration site. No large differences were found between the two strains that would point to a migration-abolishing role of the T3SS. 
Table 2 Predicted ORFs of T3SS cluster in B. terrae BS001

\begin{tabular}{|c|c|c|c|c|c|}
\hline ORF & Gene & Gene length (bp) & Protein length (aa) & Predicted function & $\begin{array}{l}\text { Homologous to B. rhizoxinica } \\
\text { HKI0450 (BLASTP) }\end{array}$ \\
\hline 1 & hpaB & 456 & 151 & Chaperone & $60 \%$ \\
\hline 2 & $s c t F$ & 222 & 73 & Needle & - \\
\hline 3 & $s c t E$ & 243 & 80 & Putative needle chaperone & $45 \%$ \\
\hline 4 & sctD & 1251 & 416 & Needle complex inner rings & $35 \%$ \\
\hline 5 & Hypothetical protein & 882 & 293 & Unknown & - \\
\hline 6 & $s c t S$ & 264 & 87 & Export apparatus & $59 \%$ \\
\hline 7 & $s c t R$ & 657 & 218 & Export apparatus & $66 \%$ \\
\hline 8 & $\operatorname{sct} Q$ & 1185 & 394 & Cytoplasmic sorting plantform & $31 \%$ \\
\hline 9 & sctP & 561 & 186 & needle length regulator & $30 \%$ \\
\hline 10 & $\operatorname{sct} V$ & 2085 & 694 & Export apparatus & $69 \%$ \\
\hline 11 & $\operatorname{sct} U$ & 1074 & 357 & Export apparatus & $52 \%$ \\
\hline 12 & $\operatorname{sct} G$ & 522 & 173 & Unknown & $42 \%$ \\
\hline 13 & sctI & 393 & 130 & Inner rod component & $36 \%$ \\
\hline 14 & sct $J$ & 801 & 266 & Needle complex inner rings & $64 \%$ \\
\hline 15 & $s c t K$ & 741 & 246 & Cytoplasmic sorting platform & $36 \%$ \\
\hline 16 & $s c t L$ & 765 & 254 & Links ATPase to sorting platform & $45 \%$ \\
\hline 17 & $\operatorname{sct} N$ & 1359 & 452 & ATPase & $77 \%$ \\
\hline 18 & $\operatorname{sct} O$ & 498 & 165 & Unknown & $38 \%$ \\
\hline 19 & $\operatorname{sct} T$ & 858 & 285 & Export apparatus & $54 \%$ \\
\hline 20 & Hypothetical protein & 429 & 142 & Unknown & - \\
\hline 21 & $H r p B$ & 1455 & 484 & Unknown & $44 \%$ \\
\hline 22 & $\operatorname{sct} C$ & $>646$ & $>215$ & Needle complex outer rings & N.A. \\
\hline
\end{tabular}

N.A. not applicable since the sequence of $s c t C$ in BS001 is not completed $s c t D$ : $s c t D$ gene was knocked out to construct T3SS mutant strain

We thus surmised that more subtle effects of the sctD mutation might only become visible when using mutant and wild-type cells in direct competition with each other.
Joint inoculations Upon joint introduction, at the inoculation site, the proportion of the mutant strain in the mix remained at around $50 \%$, i.e., $48.7 \pm 7.3 \%$ at day 0 to $45.3 \pm 3.6 \%$ at day

Fig. 2 Growth curves of

B. terrae BS001 and B. terrae $\mathrm{BS} 001 \Delta s c t D$ in LB broth medium

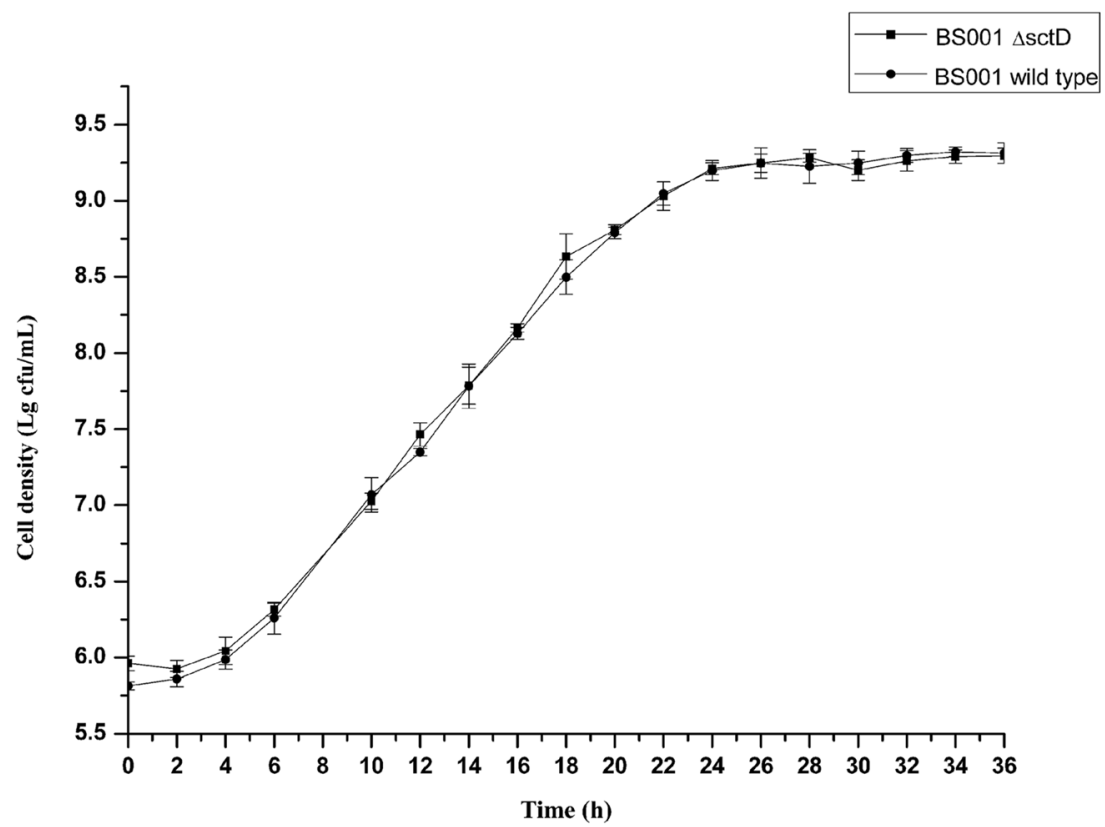



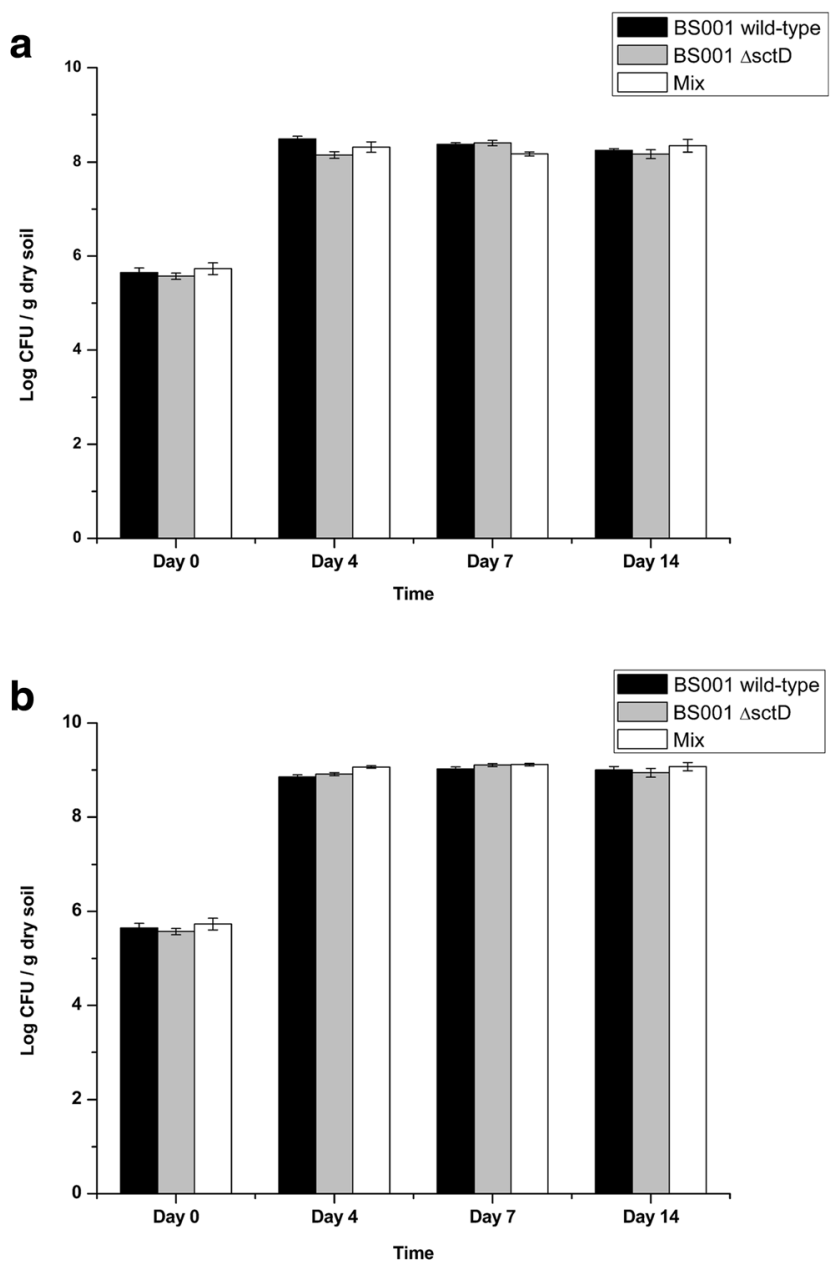

C

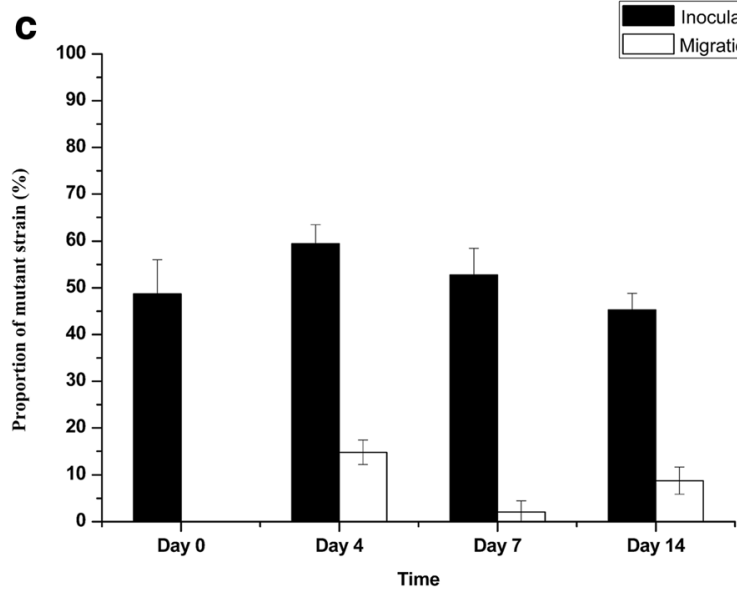

Fig. 3 Population dynamics of bacterial cells in mycosphere of Lyophyllum sp. strain Karsten. a Cell abundance (as indicated by editor) at inoculation site. $\mathbf{b}$ Cell abundance at migration site. $\mathbf{c}$ Abundance of mutant strain in the mixed-inoculant experiment in the mycosphere

14. However, at the migration site, this proportion decreased to $14.8 \pm 2.6 \%$ at day 4 and $2.5 \pm 2.4 \%$ at day 7 , after which it increased slightly to $8.8 \pm 2.9 \%$ at day 14 (Fig. 3c). These values were all significantly lower than the initial $48.7 \pm 7.3 \%$
$(P<0.001)$, as well as the further values measured at the inoculation site, suggesting that, whereas the mutant had no ecological fitness disadvantage at the older hyphae, it did so at the hyphal migration front. At the latter site, younger (growing) hyphae were prevalent.

Population dynamics of $B$. terrae BS001 in the mycosphere of T. asperellum 302

To verify whether the $\Delta s c t D$ effect reflected a "generalist" type of interaction of strain BS001 with fungal surfaces, we extended our study to the mycosphere of T. asperellum 302 . T. asperellum 302 is a biocontrol fungus with which $B$. terrae BS001 has previously been found to form a migratory association (Nazir et al. 2014). Since this organism grows very rapidly through the amended G soil, we sampled the microcosm systems at just one early time point, i.e., day 2 (Fig. 4). In separate inoculation experiments, the mutant behavior was found to be similar to that of the wild-type strain, with similar CFU abundance at the inoculation and migration sites between the two strains, reaching up to $10^{8} \mathrm{CFU} / \mathrm{g}$ dry soil (Fig. 4a). In the microcosms inoculated with (1:1) mixed cultures, the proportion of the mutant strain at the inoculation site remained at the initial level, i.e., around $59.3 \pm 5.2 \%$. In contrast, it decreased significantly $(P<0.01)$, to $18.0 \pm$ $10.4 \%$, at the migration site (day 2; Fig. 4b). These data are in line with those obtained with Lyophyllum sp. strain Karsten, indicating that also the T. asperellum 302 growing hyphal front selectively carried more wild-type (T3SS-positive) than mutant (T3SS-negative) cells through the soil.

\section{Discussion}

Fungal mycelium developing in soil allows bacterial cells to move to new microhabitats in which local nutrients can be utilized (Bravo et al. 2013), "remote" pollutants can be accessed (Wick et al. 2007; Zhao et al. 2016) and survival can be supported (Warmink and van Elsas 2009). Additionally, in plant-associated soil environments, fungal hyphae can transport bacteria, such as plant-growth-promoting or biocontrol organisms, to reach distant rhizosphere or rhizoplane sites, thus promoting effects of these (Shao et al. 2015). Then, the composition of the soil microbiome can be directed (Yu et al. 2015), rhizosphere niches, in which root-microbiome associations were disrupted, filled (Qiu et al. 2014), and phytopathogens antagonized (Tan et al. 2016). Thus, bacterial dispersal along with fungal hyphae in soil is very important for a number of key soil functions.

The T3SS has previously been suggested to play key roles in several bacterial-plant, bacterial-animal, and, incidentally, bacterial-fungal interactions (Lackner et al. 2011; Viollet et al. 2011). In this paper, we explore the role of the T3SS in the 




b

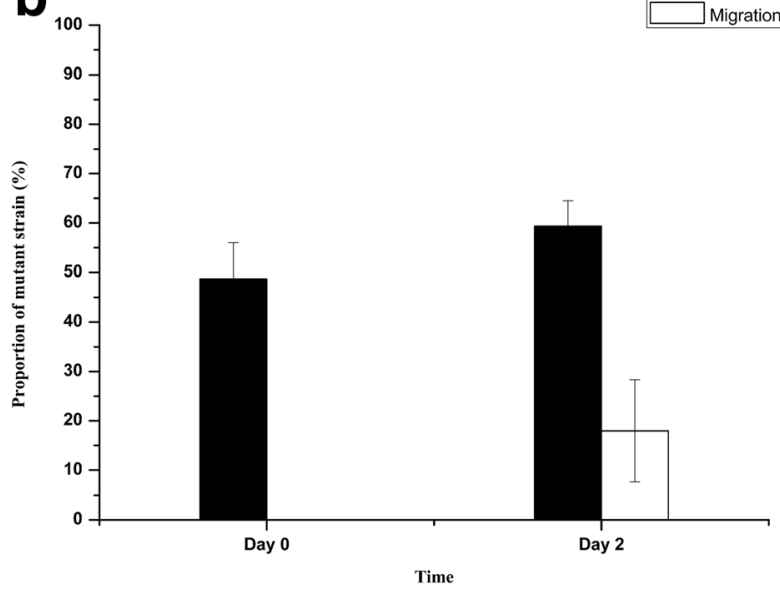

Fig. 4 Population dynamics of bacterial cells in the soil microcosm of Trichoderma asperellum 302. a Cell abundance at day 2 in the mycosphere of T. asperellum 302. b Abundance of mutant strain in the mixed-inoculant experiment in the mycosphere

interaction of the mycosphere dweller B. terrae BS001 with two saprotrophic soil fungi, Lyophyllum sp. strain Karsten and T. asperellum 302. To achieve this aim, we successfully produced an $s c t D$-minus mutant of strain BS001.

In previous work, an $s c t D$ (YscD in Yersinia pestis) mutant strain failed to secrete the effector protein YopM, indicating its key role in the T3SS (Plano and Straley 1995). Also, another sctD mutant strain ( $p r g H$, lacking a functional SpI-1 T3SS in Salmonella enterica) showed significantly lower recovery rate than the wild-type strain inside Macrosteles quadrilineatus (Dundore-Arias et al. 2015). According to the recent literature, the assembly of the T3SS has four steps, assembly of basal body rings and the export apparatus is a key first one (Burkinshaw and Strynadka 2014). SctD is a main component of the basal body ring (Burkinshaw and Strynadka 2014), linking the SctC (outer ring) and SctJ protein (component of inner ring) (Diepold et al. 2010). The subsequent recruitment of the cytoplasmic components SctK, SctL, SctN, SctO, and SctQ, and their linkage to the basal body, are thought to be dependent on the first step (Galan et al. 2014). Thus, SctD plays a key role in the assembly of the T3SS, and we predict that a functional T3SS complex is not being built in the absence of SctD in B. terrae BS001 $\Delta s c t D$.

One key requirement for sound mutant effect testing is that it did not undergo any other changes than the intended one. As far as we could see, this was the case for strain BS001 $\Delta s c t D$, as it showed similar growth dynamics to the wild-type strain and did not reveal any capability to utilize the substrates encompassed in the BIOLOG GEN III plates (columns 1-9). Moreover, there was no impact of the mutation on the capacity of the strain to deal with stress (BIOLOG GEN III plates, columns 10-12). These data demonstrate that, within the confines of the experiment, the absence of the functional sctD gene (affecting the stability of the T3SS) had no significant effect on bacterial development and physiological status. In the sterilized soil, nutrients were expected to be available, first as a result of the autoclaving (releasing microbial based nutrients, Juarez et al. 2013) and secondly as a result of the release by active fungal hyphae. Thus, growth of bacterial cells in soil, along with the developing fungal hyphae, was expected, resulting in the increases of cell abundance from about $10^{5} \mathrm{cfu} / \mathrm{g}$ dry soil at day 0 to $10^{8} \mathrm{cfu} / \mathrm{g}$ dry soil at day 4 .

We previously obtained circumstantial evidence for the contention that the T3SS may be involved in the B. terrae BS001-fungal interaction (Warmink and van Elsas 2008; 2009). In the current study, we provide direct evidence for this contention, on the basis of the data obtained with two fungi in coinoculation experiments. Thus, when both wild-type and mutant strains were introduced together, in a ratio close to $1: 1$, in the soil microcosms, the wild-type strain clearly outcompeted the mutant in terms of comigration with both fungal counterparts. However, this effect was not observed at the inoculation site, where mutant/wild-type ratio's remained roughly stable. Thus, the T3SS clearly plays a role, albeit a relatively minor one, in the migratory interaction of B. terrae BS001 with the two soil fungi. In a recent study, the NF-T3SS was considered to be derived from the flagellar T3SS, with a complex evolutionary history (Abby and Rocha 2012). However, in our study with B. terrae BS001, the loss of the functional T3SS (sctD gene, in this case) did not incur any differential motility toward fungal hyphae between the wild-type and mutant strain (Haq et al. 2016). On the contrary, the $B$. terrae BS001 $\Delta s c t D$ mutant strain adhered to a lower extent to Lyophyllum sp. strain Karsten surfaces than the wild-type strain, with a potential involvement of ceramide monohexoside $(\mathrm{CMH})$ in the binding (Haq et al. 2016). These novel data demonstrated that the T3SS can serve as an adhesion-enhancing mechanism, mediating or strengthening the attachment of bacterial cells to a host cell surface. Thus, the wild-type strain most likely outcompeted the mutant strain in the comigrational behavior by occupying more binding sites or by strengthening 
existing bonds at the surfaces of the fungal tips. The enhanced number of wild-type versus mutant cells adhering to the extending fungal hyphae thus is at the basis of the detected mutant/wild-type ratio shifts.

The fact that the mutant strain apparently comigrated, to a lesser extent, with the growing fungal hyphae indicated that the comigration was not solely mediated by the T3SS. In other words, the comigration process is probably multifaceted, having the involvement of other (adhesion and/or movement) mechanisms, which jointly result in a superior migration ability. Examination of the 11.5 Mb BS001 genome, with 12,047 predicted coding sequences (CDSs) (Haq et al. 2014) indicated a suite of other systems that possibly mediate the adhesion of bacterial cell to host cell surfaces, i.e., systems for biofilm formation, type 4 pili, flagella, and type 4 and type 6 secretion systems (T4SS, T6SS). Type 4 pili and flagella are not only motility appendages, but they can also act as adhesins that connect bacteria to surfaces and facilitate near-surface motility prior to biofilm formation (Conrad 2012; Laverty et al. 2014). The T4SS is required in conjugation, which is cell-to-cell contact dependent. In this respect, transfers of conjugative plasmids from bacteria to fungi have been reported (Zhang et al. 2014). Additionally, in some cases, the T4SS is responsible for secretion of DNA into the external milieu, improving biofilm formation (Zweig et al. 2014). There are three T4SS gene clusters on the genome of $B$. terrae BS001. Only one of them, which is present on a 70.42-kb genomic island, contains all canonical T4SS functions (Haq et al. 2014). We assume this system may mediate $B$. terrae BS001 adhesion to the fungal surface and/or promote biofilm formation around the fungal hyphae indirectly by releasing DNA to its surroundings. Moreover, B. terrae BS001 carries several gene clusters that encode extracellular polysaccharide biosynthesis, including poly-beta-1, 6-N-acetyl-D-glucosamine (PGA) and Pel (a glucose-rich polysaccharide polymer) production (Haq et al. 2014). In other studies, these polymers have been shown to be able to aid biofilm formation (Vasseur et al. 2005; Itoh et al. 2008). Furthermore, cellulose has been reported to be involved in Salmonella biofilm formation on Aspergillus niger (Brandl et al. 2011), and cellulose production loci are also present in the B. terrae BS001 genome. From these considerations, we surmise that the migratory association of $B$. terrae BS001 with soil fungi is a multifaceted process, and the presence of the T3SS is an asset that enhances the migratory ability but is not strictly necessary.

In conclusion, this study confirms the role of a bacterial T3SS as a comigration helper mechanism through soil in the mycosphere of two soil fungi. B. terrae BS001 cells thus use their T3SS as helper systems that promote their migration in the mycosphere. Finally, we did not address the potential of the T3SS to deliver effector molecules into fungal cells, as our primary aim was to address the overall effect on migration. Future studies might address the potential fungal physiology modulatory role of the strain BS001 T3SS. Our data are consistent with the contention that, besides the T3SS, other surface-exposed systems are employed by $B$. terrae BS001 in its interaction with soil fungi. Thus, strains debilitated in, for instance, type 4 pili (T4P), flagella, the T4SS, or the T6SS should be constructed for a further understanding the mechanism behind bacterial migration along with fungal hyphae.

Acknowledgments $\mathrm{Pu}$ Yang, Miaozhi Zhang and Miao Wang were financially supported by the Chinese Scholarship Council (CSC).

Open Access This article is distributed under the terms of the Creative Commons Attribution 4.0 International License (http:// creativecommons.org/licenses/by/4.0/), which permits unrestricted use, distribution, and reproduction in any medium, provided you give appropriate credit to the original author(s) and the source, provide a link to the Creative Commons license, and indicate if changes were made.

\section{References}

Abby SS, Rocha EP (2012) The non-flagellar type III secretion system evolved from the bacterial flagellum and diversified into host-cell adapted systems. PLoS Genet 8:e1002983. doi:10.1371/journal. pgen. 1002983

Brandl MT, Carter MQ, Parker CT, Chapman MR, Huynh S, Zhou Y (2011) Salmonella biofilm formation on Aspergillus niger involves cellulose-chitin interactions. PLoS ONE 6:e25553. doi:10.1371 /journal.pone.0025553

Bravo D, Cailleau G, Bindschedler S, Simon A, Job D, Verrecchia E, Junier P (2013) Isolation of oxalotrophic bacteria able to disperse on fungal mycelium. FEMS Microbiol Lett 348:157-166

Burkinshaw BJ, Strynadka NC (2014) Assembly and structure of the T3SS. Biochim Biophys Acta 1843:1649-1663

Conrad JC (2012) Physics of bacterial near-surface motility using flagella and type IV pili: implications for biofilm formation. Res Microbiol 163:619-629

Correa VR, Majerczak DR, Ammar EI-D, Merighi M, Pratt RC, Hogenhout SA, Coplin DL, Redinbaugh MG (2012) The bacterium Pantoea stewartii uses two different type III secretion systems to colonize its plant host and insect vector. Appl Environ Microbiol 78: $6327-6336$

Cusano AM, Burlinson P, Deveau A, Vion P, Uroz S, Preston GM, GreyKlett P (2011) Pseudomonas fluorescens BBc6R8 type III secretion mutants no longer promote ectomycorrhizal symbiosis. Environ Microbiol Rep 3:203-210

Dai WJ, Zeng Y, Xie ZP, Staehelin C (2008) Symbiosis-promoting and deleterious effects of NopT, a novel type 3 effector of Rhizobium sp. strain NGR234. J Bacteriol 190:5101-5110

Delahay RM, Shaw RK, Elliott SJ, Kaper JB, Knutton S, Frankel G (2002) Functional analysis of the enteropathogenic Escherichia coli type III secretion system chaperone CesT identifies domains that mediate substrate interactions. Mol Microbiol 43:61-73

Diepold A, Amstutz M, Abel S, Sorg I, Jenal U, Cornelis GR (2010) Deciphering the assembly of the Yersinia type III secretion injectisome. EMBO J 29:1928-1940

Diepold A, Wiesand U, Cornelis GR (2011) The assembly of the export apparatus (YscR, S, T, U, V) of the Yersinia type III secretion apparatus occurs independently of other structural components and involves the formation of an $\mathrm{YscV}$ oligomer. Mol Microbiol 82: $502-514$ 
Diepold A, Kudryashev M, Delalez NJ, Berry RM, Armitage JP (2015) Composition, formation, and regulation of the cytosolic c-ring, a dynamic component of the type III secretion injectisome. PLoS Biol 13:e1002039. doi:10.1371/journal.pbio.1002039

Dundore-Arias JP, Groves RL, Barak JD (2015) Influence of $p r g H$ on the persistence of ingested Salmonella enterica in the leafhopper Macrosteles quadrilineatus. Appl Environ Microbiol 81:6345-6354

Galan JE, Lara-Tejero M, Marlovits TC, Wagner S (2014) Bacterial type III secretion systems: specialized nanomachines for protein delivery into target cells. Annu Rev Microbiol 68:415-438

Haq IU, Graupner K, Nazir R, van Elsas JD (2014) The genome of the fungal-interactive soil bacterium Burkholderia terrae BS001-a plethora of outstanding interactive capabilities unveiled. Genome Biol Evol 6:1652-1668

Haq IU, da Rocha Calixto RO, Yang P, dos Santos GM, Barreto-Bergter E, van Elsas JD (2016) Chemotaxis and adherence to fungal surfaces are key components of the behavioral response of Burkholderia terrae BS001 with two selected soil fungi. FEMS Microbiol Ecol. doi:10.1093/femsec/fiw164

Hoiczyk E, Blobel G (2001) Polymerization of a single protein of the pathogen Yersinia enterocolitica into needles punctures eukaryotic cells. PNAS 98:4669-4674

Itoh Y, Rice JD, Goller C, Pannuri A, Taylor J, Meisner J, Beveridge TJ, Preston JF 3rd, Romeo T (2008) Roles of pgaABCD genes in synthesis, modification, and export of the Escherichia coli biofilm adhesin poly-beta-1,6-N-acetyl-D-glucosamine. J Bacteriol 190: 3670-3680

Jackson MW, Plano GV (2000) Interactions between type III secretion apparatus components from Yersinia pestis detected using the yeast two-hybrid system. FEMS Microbiol Lett 186:85-90

Journet L, Agrain C, Broz P, Cornelis GR (2003) The needle length of bacterial injectisomes is determined by a molecular ruler. Science 302:1757-1760

Juarez S, Nunan N, Duday AC, Pouteau V, Chenu C (2013) Soil carbon mineralisation responses to alterations of microbial diversity and soil structure. Biol Fertil Soils 49:929-948

Lackner G, Moebius N, Hertweck C (2011) Endofungal bacterium controls its host by an hrp type III secretion system. ISME J 5:252-261

Laverty G, Gorman SP, Gilmore BF (2014) Biomolecular mechanisms of Pseudomonas aeruginosa and Escherichia coli biofilm formation. Pathogens 3:596-632

Nazir R, Zhang M, de Bore W, van Elsas JD (2012) The capacity to comigrate with Lyophyllum sp. strain Karsten through different soils is spread among several phylogenetic groups within the genus Burkholderia. Soil Biol Biochem 50:221-233

Nazir R, Warmink JA, Voordes DC, van de Bovenkamp HH, van Elsas JD (2013) Inhibition of mushroom formation and induction of glycerol release-ecological strategies of Burkholderia terrae BS001 to create a hospitable niche at the fungus Lyophyllum sp. strain Karsten. Microb Ecol 65:245-254

Nazir R, Tazetdinova DI, van Elsas JD (2014) Burkholderia terrae BS001 migrates proficiently with diverse fungal hosts through soil and provides protection from antifungal agents. Front Microbiol 5: 598. doi:10.3389/fmicb.2014.00598

Neeld D, Jin Y, Bichsel C, Jia J, Guo J, Bai F, Wu W, Ha UH, Terada N, Jin S (2014) Pseudomonas aeruginosa injects NDK into host cells through a type III secretion system. Microbiology 160:1417-1426

Okazaki S, Zehner S, Hempel J, Lang K, Gottfert M (2009) Genetic organization and functional analysis of the type III secretion system of Bradyrhizobium elkanii. FEMS Microbiol Lett 195:88-95

Piromyou P, Songwattana P, Greetatorn T, Okubo T, Kakizaki KC, Prakamhang J, Tittabutr P, Boonkerd N, Teaumroong N, Minamisawa K (2015) The type III secretion system (T3SS) is a determinant for rice-endophyte colonization by non-photosynthetic Bradyrhizobium. Microbe Environ 30:291-300
Plano GV, Schesser K (2013) The Yersinia pestis type III secretion system: expression, assembly and role in the evasion of host defenses. Immunol Res 57:237-245

Plano GC, Straley SC (1995) Mutations in $y s c C$, yscD, and yscG prevent high-level expression and secretion of $\mathrm{V}$ antigen and Yops in Yersinia pestis. J Bacteriol 177:3843-3854

Qiu M, Li S, Zhou X, Cui X, Vivanco JM, Zhang N, Shen Q, Zhang R (2014) De-coupling of root-microbiome associations followed by antagonist inoculation improves rhizosphere soil suppressiveness. Biol Fertil Soils 50:217-224

Rezzonico F, Binder C, Defago G, Moenne-Loccoz Y (2005) The type III secretion system of biocontrol Pseudomonas fluorescens KD targets the phytopathogenic Chromista Pythium ultimum and promotes cucumber protection. Mol Plant Microbe Interact 18:991-1001

Saad MM, Crevecoeur M, Masson-Boivin C, Perret X (2012) The type 3 protein secretion system of Cupriavidus taiwanensis strain LMG19424 compromises symbiosis with Leucaena leucocephala. Appl Environ Microbiol 78:7476-7479

Shao J, Xu Z, Zhang N, Shen Q, Zhang R (2015) Contribution of indole-3acetic acid in the plant growth promotion by the rhizospheric strain Bacillus amyloliquefaciens SQR9. Biol Fertil Soils 51:321-330

Silver AC, Kikuchi Y, Fadl AA, Sha J, Chopra AK, Graf J (2007) Interaction between innate immune cells and a bacterial type III secretion system in mutualistic and pathogenic associations. PNAS 104:9481-9486

Simon R, Priefer U, Puhler A (1983) A broad host range mobilization system for in vivo genetic engineering: transposon mutagenesis in gram negative bacteria. Nat Biotechnol 1:784-791

Tan S, Gu Y, Yang C, Dong Y, Mei X, Shen Q, Xu Y (2016) Bacillus amyloliquefaciens T-5 may prevent Ralstonia solanacearum infection through competitive exclusion. Biol Fertil Soils 52:341-351

Torruellas J, Jackson MW, Pennock JW, Plano GV (2005) The Yersinia pestis type III secretion needle plays a role in the regulation of Yop secretion. Mol Microbiol 57:1719-1733

Vasseur P, Vallet-Gely I, Soscia C, Genin S, Filloux A (2005) The pel genes of the Pseudomonas aeruginosa PAK strain are involved at early and late stages of biofilm formation. Microbiology 151:985-997

Viollet A, Corberand T, Mougel C, Robin A, Lemanceau P, Mazurier S (2011) Fluorescent pseudomonads harboring type III secretion genes are enriched in the mycorrhizosphere of Medicago truncatula. FEMS Microbiol Ecol 75:457-467

Warmink JA, van Elsas JD (2008) Selection of bacterial populations in the mycosphere of Laccaria proxima: is type III secretion involved? ISME J 2:887-900

Warmink JA, van Elsas JD (2009) Migratory response of soil bacteria to Lyophyllum sp. strain Karsten in soil microcosms. Appl Environ Microbiol 75:2820-2830

Warmink JA, Nazir R, Corten B, van Elsas JD (2011) Hitchhikers on the fungal highway: the helper effect for bacterial migration via fungal hyphae. Soil Biol Biochem 43:760-765

Wick LY, Remer R, Wurz B, Reichenbach J, Braun S, Schafer F, Harms H (2007) Effect of fungal hyphae on the access of bacteria to phenanthrene in soil. Environ Sci Technol 41:500-505

Wood SE, Jin J, Lloyd SA (2008) YscP and YscU switch the substrate specificity of the Yersinia type III secretion system by regulating export of the inner rod protein YscI. J Bacteriol 190:4252-4262

Yip CK, Strynadka NC (2006) New structural insights into the bacterial type III secretion system. Trends Biochem Sci 31:223-230

Yu Z, Zhang Y, Luo W, Wang Y (2015) Root colonization and effect of biocontrol fungus Paecilomyces lilacinus on composition of ammonia-oxidizing bacteria, ammonia-oxidizing archae and fungal populations of tomato rhizosphere. Biol Fertil Soils 51:343-351

Zhang M, Pereira e Silva Mde C, Chaib De Mares M, van Elsas JD (2014) The mycosphere constitutes an arena for horizontal gene transfer with strong evolutionary implications for bacterial-fungal interactions. FEMS Microbiol Ecol 89:516-526 
Zhao H, Du H, Feng N, Xiang L, Li Y, Li H, Cai Q, Mo C (2016) Biodegradation of di- $n$-butylphthalate and phthalic acid by a novel Providencia sp. 2D and its stimulation in a compost-amended soil. Biol Fertil Soils 52:65-76
Zweig M, Schork S, Koerdt A, Siewering K, Sternberg C, Thormann K, Albers SV, Molin S, van der Does C (2014) Secreted single-stranded DNA is involved in the initial phase of biofilm formation by Neisseria gonorrhoeae. Environ Microbiol 16:1040-1052 